\title{
Contribution of pelvic and para-aortic lymphadenectomy with sentinel node biopsy in patients with IB2-IIB cervical cancer
}

\author{
E Chéreau*,', J-G Feron', M Ballester', C Coutant', C Bezu', R Rouzier', E Touboul ${ }^{2}$ and E Daraï' \\ 'Department of Gynecology-Obstetrics, Hôpital Tenon, Assistance Publique des Hôpitaux de Paris, CancerEst, Université Pierre et Marie Curie Paris 6, \\ 4 rue de la Chine, Paris 75020, France; ${ }^{2}$ Department of Radiotherapy, Hôpital Tenon, Assistance Publique des Hôpitaux de Paris, CancerEst, Université \\ Pierre et Marie Curie Paris 6, Paris 75020, France
}

\begin{abstract}
OBJECTIVE: Detection of lymph node involvement in women with IB2-IIB cervical cancer could have a positive effect on survival. We set out to evaluate the incidence of pelvic and/or para-aortic lymph node involvement using the sentinel node (SN) biopsy and its impact on survival.

METHODS: From 2002 to 20 10, 66 women with IB2-IIB cervical cancer underwent a pelvic and paraaortic lymphadenectomy with SN biopsy. Survival between groups according to lymph node status was evaluated.

RESULTS: Mean tumour size was $43.5 \mathrm{~mm}$. At least one SN was detected in 69\% of the 45 SN procedures performed. Sixteen of these patients had metastatic SN and the false negative rate was 20\%. Metastatic pelvic SNs or non-SNs were detected in 33 patients (50\%), including pelvic-positive nodes in 26 (40\%), pelvic- and paraaortic-positive lymph nodes in seven (1 I\%), and paraaortic skip metastases in two (6\%). Positive paraaortic node was the sole determinant for disease-free survival (DFS) and overall survival (OS; $P<0.001)$. Differences in DFS and OS between groups according to the nodal status were observed $(P<0.00 \mathrm{I})$.

CONCLUSION: SN procedure gave a higher rate of metastasis detection. Further studies are required to evaluate whether pre-therapeutic node staging, including paraaortic and pelvic lymphanedectomy, should be performed.
\end{abstract}

British Journal of Cancer (2012) 106,39-44. doi:10.1038/bjc.201 I.54I www.bjcancer.com

Published online 6 December 2011

(c) 2012 Cancer Research UK

Keywords: pelvic lymphadenectomy; paraaortic lymphadenectomy; locally advanced cervical cancer; sentinel node biopsy; survival

Despite a recent revision of the FIGO (International Federation of Gynecology and Obstetrics) classification, cervical cancer continues to be the only gynaecological malignancy that is not surgically staged (Petru et al, 2009). This contributes to difficulties in evaluating the effect of therapy, particularly of lymphadenectomy, on survival for locally advanced stages of cervical cancer (higher than or equal to stage IB2). Indeed, lymph node involvement is relatively frequent in locally advanced stages of cervical cancer and is a major determinant for adjuvant therapy (Zander et al, 1981; Shepherd, 1996; Morice and Castaigne, 2005).

Imaging techniques including CT, MRI and PET have a high diagnostic accuracy for evaluating enlarged lymph nodes, but a poor accuracy for regular-sized lymph nodes (Hricak et al, 1988; Kim et al, 1993, 1994; Subak et al, 1995; Boss et al, 2000; Sheu et al, 2001; Hertel et al, 2002; Narayan et al, 2003; Kamelle et al, 2004; Marnitz et al, 2005; Selman et al, 2008). Systematic lymphadenectomy is thus recommended to evaluate metastases in pelvic and/or para-aortic lymph nodes (PALNs) (Zander et al, 1981; Piver, 1984; Lanciano et al, 1991; Chu et al, 1997; Michel et al, 1998; Stryker and Mortel, 2000; Vergote et al, 2002; Narayan et al, 2003). Sentinel node (SN) biopsy has also become widespread to

*Correspondence: Dr E Chéreau; E-mail: elisabeth.chereau@gmail.com Received 26 August 2011; revised 7 November 2011; accepted 8 November 201 I; published online 6 December 201 I determine lymph node status in early stages of cervical cancer (Selman et al, 2008; Altgassen et al, 2009). However, the interest of the SN biopsy in locally advanced stages of cervical cancer is more debatable, because of the low SN detection rate and high false negatives (Barranger et al, 2003, 2004a,b; Coutant et al, 2007; Altgassen et al, 2009).

Although a prospective study has shown a survival disadvantage for patients following surgical staging compared with clinical staging when concurrent radiochemotherapy (CRC) is recommended (Lai et al, 2003), most authors agree that lymph node status should be assessed by systematic lymphadenectomy. However, a debate exists whether paraaortic lymphadenectomy alone is sufficient or whether a pelvic and paraaortic lymphadenectomy should be performed systematically. Leblanc et al (2007) recommended a paraaortic lymphadenectomy alone, considering that CRC possibly associated with localised boost on positive pelvic nodes and/or on the parametria are sufficient to control local regional disease. In contrast, Houvenaeghel et al (2006) demonstrated persistence of active pelvic lymph node metastases after CRC, and that pelvic lymphadenectomy could reduce the rate of lateropelvic recurrences whatever the PALN status.

Therefore, the aim of the present retrospective study was to evaluate the incidence of pelvic and/or PALN involvement, using both SN biopsy and systematic pelvic and paraaortic lymphadenectomy, and the impact on survival in women with advanced stages of cervical cancer (stage IB2 or II). 
40

\section{PATIENTS AND METHODS}

\section{Patients}

From 2002 to 2010, 66 women with locally advanced cervical cancer corresponding to 1988 FIGO stage IB2 or II underwent a pre-therapeutic pelvic and paraaortic lymphadenectomy by laparoscopy in the gynaecology unit of Tenon Hospital, France (Barranger et al, 2003, 2004a, b; Coutant et al, 2007). All the women had biopsy-proven cervical cancer and had undergone pelvic MRI, and 45 of the 66 women had undergone a laparoscopic SN procedure before pelvic and paraaortic lymphadenectomy.

All women gave informed written consent to the therapeutic procedures and to the analysis of data related to their malignancy in accordance with institutional guidelines and the Declaration of Helsinki. The protocol was approved by the local Ethics Committee.

The medical records were reviewed to determine age, the body mass index, tumour stage, histology, tumour size on MRI, surgical procedure, intra- and postoperative complications, and the final pelvic and paraaortic node status. Outcome was obtained from the outpatient records.

The predictive factors for disease-free survival (DFS) and overall survival (OS) were analysed in univariate and multivariate analysis to provide survival data. Survival between groups according to their nodal histological status - positive or negative pelvic nodes and positive or negative paraaortic nodes - was evaluated.

\section{Technique}

SN procedure Ths SN procedure was performed as previously reported (Barranger et al, 2003; Coutant et al, 2007). The pelvic and lower paraaortic regions were carefully inspected by laparoscopy for lymph ducts and dye uptake by lymph nodes. All blue and/or hot lymph nodes were removed separately. The position of each $\mathrm{SN}$ relative to the major pelvic vessels, vena cava or aorta was recorded.

After the SN procedure, systematic transperitoneal lymph node dissection extending from the external iliac (and obturator nerve) to the level of the left renal vein was performed. The absence of residual pelvic or paraaortic radioactivity was verified before and after pelvic and paraaortic lymphadenectomy.

Lymph nodes with macroscopic metastases were sectioned. Normal-appearing SNs were cut perpendicular to the long axis. All SNs were submitted to intra-operative imprint cytology. Air-dried cytological smears were prepared by scraping the cut surfaces and staining with a rapid May-Grünwald-Giemsa method. Each half$\mathrm{SN}$ was sectioned at 3-mm intervals. Each 3-mm section was analysed at four additional levels of $150 \mu \mathrm{m}$ and four parallel sections; one was used for hematoxylin and eosin (H\&E) staining, and H\&E-negative sections were examined by immunohistochemistry (IHC) with an anticytokeratin antibody cocktail (cytokeratins AE1-AE3; Dako Corporation, Glostrup, Denmark). Non-SNs were submitted totally and blocked individually after 3-mm sectioning and $\mathrm{H} \& \mathrm{E}$ staining.

Macrometastases was defined by a single focus of metastatic disease per node measuring more than $2 \mathrm{~mm}$, micrometastases as a focus of metastatic disease ranging from $0.2 \mathrm{~mm}$ to no more than $2 \mathrm{~mm}$ and, in accordance with previous studies (Marchiole et al, 2005; Bezu et al, 2010), submicrometastases as metastases measuring no more than $0.2 \mathrm{~mm}$ including the presence of a single non-cohesive tumour cell. SNs and non-SNs were considered positive when they contained macrometastases, micrometastases or submicrometastases.

\section{Concurrent radiochemotherapy}

External pelvic radiation therapy was given through four orthogonal fields: antero-posterior (AP) and postero-anterior (PA), and two lateral fields. The upper limit of the AP/PA field was L4-L5 interspace. The lower limit extended distally to the midportion of the obturator foramen or the lowest level of disease with a $3-\mathrm{cm}$ margin, and laterally $2 \mathrm{~cm}$ beyond the lateral margins of the bony pelvic wall. The upper and lower limits of the lateral fields were the same as those of the AP/PA field. The anterior limit of the lateral field was a horizontal line drawn at the anterior border of the pubic symphysis. The posterior limit of lateral field was placed at the S2-S3 interspace. Customised blocks were used to spare the anterior half of the rectum posteriorly and a proportion of small bowel anteriorly.

Pelvic radiation therapy consisted of $40 \mathrm{~Gy}$, using $2.25 \mathrm{~Gy}$ per fraction, 4 days a week. A vaginal booster dose of $20 \mathrm{~Gy}$ was given at 5-6 weeks by means of brachytherapy. Brachytherapy was performed after radical hysterectomy when uterine catherisation was impossible.

Concurrent chemotherapy was given during the 1st and 4th week of radiation therapy and consisted of a continuous 5 -fluorouracil infusion ( $750 \mathrm{mg} \mathrm{m}^{-2}$ per day) and a cisplatin bolus $\left(20-25 \mathrm{mg} \mathrm{m}^{-2}\right.$ per day) $1 \mathrm{~h}$ before radiotherapy, for days $1,2,4$ and 5 . When the pelvic and paraaortic nodes were not involved, simple or radical laparoscopic hysterectomy was performed 6 weeks after the end of CRC. Women with positive lymph node involvement underwent a specific CRC regimen. For these women, the total dose of external radiotherapy delivered was $45 \mathrm{~Gy}$ with an iliac boost of $10 \mathrm{~Gy}$, followed by the same brachytherapy regimen. The chemotherapy protocol was the same, but delivered the 1st and the 5 th week of irradiation.

Patients with positive aortic nodes received extended-field radiation up to the level of T12-L1. The lateral limits were set $4 \mathrm{~cm}$ from the midline.

\section{Statistical analysis}

Data were analysed using the $\chi^{2}$-test or the Fisher's exact test and the Student's $t$-test. Differences were considered significant when $P<0.05$. OS time was calculated in months from the date of surgery to death, or the date of last follow-up for surviving patients and DFS time from the date of surgery to recurrence. The KaplanMeier method was used to estimate the survival distribution, and comparisons of survival were made by the use of the log-rank test. Cox proportional hazards regression was used for multivariate analysis. Informative prognostic factors for outcome were selected according to Akaike Information Criteria.

\section{RESULTS}

\section{Epidemiological and surgical characteristics of the population}

Patient and tumour characteristics are reported in Table 1. The mean tumour size was $43.5 \mathrm{~mm}$ (range: 12-70). Eighty-six percent of the patients had a squamous cell carcinoma. About half of the patients had a moderately or poorly differentiated carcinoma, and more than half of the patients had FIGO stage IIB.

All 66 patients underwent both pelvic and paraaortic lymphadenectomy for a cervical cancer. This procedure was followed by CRC and brachytherapy for 58 of them (88\%). A total of 2 of the 66 patients required a conversion to laparotomy: for anaesthesiological disorders related to hypoventilation for one and for ureteral injury requiring a bladder reimplantation for the other. No bleeding or vascular injury requiring laparotomy was observed. Moreover, none of these patients had unresectable bulky nodes. Eight patients (12\%) with stage II A cervical cancer and tumour size below $4 \mathrm{~cm}$ underwent a laparoscopic pelvic and paraaortic lymphadenectomy associated with a radical hysterectomy during the same surgical procedure, followed by CRC and brachytherapy. 
Table I Epidemiological and surgical characteristics of the 66 patients with locally advanced stages of cervical cancers, who underwent pelvic and para-aortic lymphadenectomy and sentinel lymph node biopsy

\begin{tabular}{|c|c|}
\hline Characteristics & $\begin{array}{l}\text { Patients } \\
(n=66)\end{array}$ \\
\hline Mean age in years (range) & $48.8(28-76)$ \\
\hline Post-menopausal patients (\%) & $26(39)$ \\
\hline Mean body mass index, $\mathrm{kg} \mathrm{m}^{-2}$ (range) & $23.4(16.8-35.0)$ \\
\hline Mean tumour size on MRI, mm (range) & $43.5(12-70)$ \\
\hline \multicolumn{2}{|l|}{ Tumour location in the cervix } \\
\hline Ectocervical (\%) & $62(94)$ \\
\hline Endocervical (\%) & $3(5)$ \\
\hline Exo and endocervical (\%) & I (I) \\
\hline \multicolumn{2}{|l|}{ Tumour histology } \\
\hline Squamous cell carcinoma (\%) & $57(86)$ \\
\hline Adenocarcinoma (\%) & $9(14)$ \\
\hline \multicolumn{2}{|l|}{ Histological grade of the tumour } \\
\hline Well differentiated (\%) & $30(45)$ \\
\hline Moderately differentiated (\%) & $10(15)$ \\
\hline Poorly differentiated (\%) & $15(23)$ \\
\hline Unclassified (\%) & $11(17)$ \\
\hline \multicolumn{2}{|l|}{ FIGO classification } \\
\hline IB2 (\%) & $23(34)$ \\
\hline IIA (\%) & $8(12)$ \\
\hline IIB (\%) & $35(54)$ \\
\hline \multicolumn{2}{|l|}{ Therapy associated with LPPAL } \\
\hline Chemoradiotherapy and brachytherapy (\%) & $27(4 I)$ \\
\hline $\begin{array}{l}\text { Chemoradiotherapy and brachytherapy, followed by } \\
\text { hysterectomy (\%) }\end{array}$ & $31(47)$ \\
\hline $\begin{array}{l}\text { First radical hysterectomy and LPPAL, followed by } \\
\text { chemoradiotherapy and brachytherapy (\%) }\end{array}$ & $8(12)$ \\
\hline
\end{tabular}

Abbreviations: $\mathrm{FIGO}=$ International Federation of Gynecology and Obstetrics; $L P P A L=$ laparoscopic pelvic and para-aortic lymphadenectyomy; $M R I=$ magnetic resonance imaging.

\section{SN procedure}

SN procedure was performed in 45 patients, resulting in the detection of at least one SN in $69 \%$ of cases $(n=31$; Table 2$)$. A bilateral SN was found in $26 \%$ of cases $(n=12)$. The mean number of SN removed was $2.1(1-4)$ per patient. No difference in cervical cancer stages between patients with and without $\mathrm{SN}$ was detected.

Among these 45 patients, in $87 \%$ of cases (39 among 45 cases), SN was hot and blue, in 5 cases, $\mathrm{SN}$ was blue alone $(11 \%)$ and in 1 case, $\mathrm{SN}$ was radioactive alone $(2 \%)$.

Histology revealed metastatic SN in 16 patients $(52 \%, 16$ among 31 patients with at least one SN detected), including macrometastases in 12 cases and micrometastases in 4 (25\%). All positive SN were distributed in the pelvic area and had positive HES staining. Four patients had a false negative SN giving a false negative rate of $20 \%$.

\section{Pelvic and paraaortic lymphadenectomy}

The mean number of lymph nodes removed, including SNs during pelvic or paraaortic lymphadenectomy, were 12.5 nodes $(3-24)$ and 12.5 nodes (4-28), respectively. Metastatic pelvic SNs or nonSNs were detected in 33 patients $(50 \%)$. Metastatic PALNs were detected in nine patients (14\%). Skip metastases to PALNs were diagnosed in two patients in the presence of negative pelvic lymph nodes. In these two patients, the first presented $1-\mathrm{mm}$ micrometastases with one negative SN identified (HES and IHC).
Table 2 Results of the SN biopsy and of pelvic and para-aortic lymphadenectomy in 66 patients with stage IB2-IIB cervical cancer

\begin{tabular}{|c|c|}
\hline Characteristics & Number of patients (\%) \\
\hline \multicolumn{2}{|l|}{ Pelvic SN } \\
\hline Number of patients (\%) & $45(68)$ \\
\hline At least one identified SN (\%) & $31(69)$ \\
\hline Mean number of SNs per patient (range) & $2.1(1-4)$ \\
\hline Patients with bilateral SN (\%) & $12(26)$ \\
\hline \multicolumn{2}{|l|}{ Histopathology (among 3I patients with SN detected) } \\
\hline Negative SN (\%) & $15(48)$ \\
\hline Positive SN (\%) & $16(52)$ \\
\hline Macrometastasis (\%) & $12(39)$ \\
\hline Micrometastasis (\%) & $4(13)$ \\
\hline \multicolumn{2}{|l|}{ Pelvic non-SN } \\
\hline Mean number of $L N s$ per patient (range) & $12.5(3-24)$ \\
\hline \multicolumn{2}{|l|}{ Pelvic SN and non-SN } \\
\hline Number of patients & 66 \\
\hline Number of patients with metastatic LN (\%) & $33(50)$ \\
\hline SN performed (\%) & $20(60)$ \\
\hline $\mathrm{SN}+/$ non-SN+ (\%) & $7(21)$ \\
\hline $\mathrm{SN}+/$ non-SN- (\%) & $9(27)$ \\
\hline $\mathrm{SN}-/$ non-SN+ (\%) & $4(12)$ \\
\hline False negative rate $(\%)$ & $4 / 20(20)$ \\
\hline SN detection not performed (\%) & $13(40)$ \\
\hline \multicolumn{2}{|l|}{ Para-aortic lymph node } \\
\hline Number of patients & 66 \\
\hline Mean number of LNs per patient (range) & $12.5(4-28)$ \\
\hline Number of patients with metastases (\%) & $9(14)$ \\
\hline \multicolumn{2}{|l|}{ Patients with positive nodes } \\
\hline Number of patients (\%) & $35(53)$ \\
\hline Positive pelvic and paraaortic nodes (\%) & $7(20)$ \\
\hline Positive isolated pelvic nodes (\%) & $26(74)$ \\
\hline $\mathrm{SN}+/$ non-SN+ (\%) & $19(83)$ \\
\hline $\mathrm{SN}+/$ non-SN- (\%) & $7(17)$ \\
\hline $\mathrm{SN}-/$ non-SN+ & 0 \\
\hline Positive isolated para-aortic nodes (\%) & $2(6)$ \\
\hline SN performed & i \\
\hline Pelvic negative SN detected & 1 \\
\hline
\end{tabular}

Abbreviations: $\mathrm{LN}=$ lymph node; $\mathrm{SN}=$ sentinel node

The second patient did not have a SN biopsy and presented 10 pelvic nodes free from disease and one of the 28 PALNs involved.

Among the 35 patients (53\%) with positive nodes, $20 \%$ of them had both pelvic and paraaortic positives nodes, $74 \%$ had only pelvic positives nodes and $6 \%$ had isolated paraaortic positives nodes. In patients with isolated pelvic positive nodes, $83 \%$ of them had both metastatic SNs and non-SNs, whereas $17 \%$ had only metastatic SNs. None had only metastatic non-SNs.

Four false negative cases of SN procedure were observed corresponding to patients with unilateral SN detection. In three of them, only one SN was removed and in the last case, two SNs were removed. The four patients also had positive PALNs.

\section{OS and DFS}

The median follow-up was 28.3 months (2-79 months; Figures 1 and 2). Seven of the sixty-six patients (11\%) relapsed, including three centropelvic recurrences (two of three in patients without hysterectomy), two peritoneal carcinomatosis (11 and 17 months after surgery), one lateropelvic and one common iliac node recurrences. The 5-year DFS was $86 \%$ and the 5-year OS was $78 \%$. Univariable analysis for DFS found that the only significant factor was the positivity of paraaortic nodes $(P<0.001)$. This factor was 
42

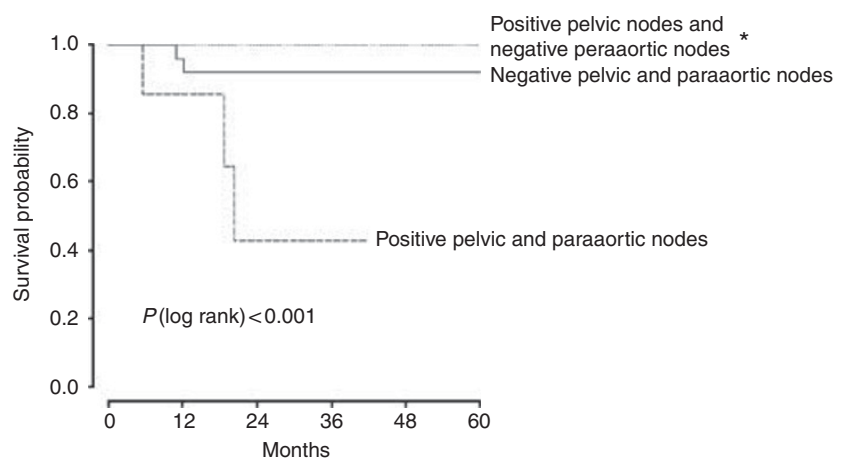

Figure I Disease-free survival according to nodal status in 66 patients with stage $\mathrm{lb} 2-\mathrm{llb}$ cervical cancer. *No significant difference in survival between patients with negative pelvic and paraaortic nodes and patients with positive pelvic nodes and negative paraaortic nodes.

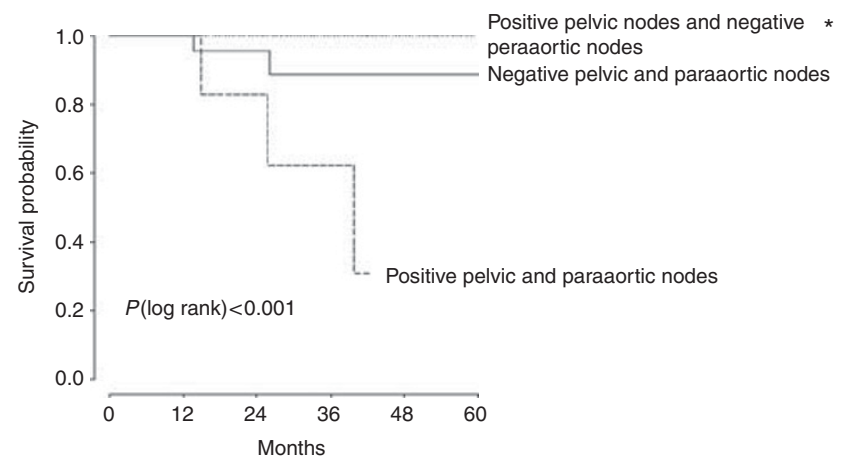

Figure 2 Overall survival according to nodal status in 66 patients with stage lb2-Ilb cervical cancer. *No significant difference in survival between patients with negative pelvic and paraaortic nodes and patients with positive pelvic nodes and negative paraaortic nodes patients.

also significant in multivariable analysis $(P=0.02)$. For OS, positive paraaortic nodes $(P<0.0001)$ remained the sole determinant factor in univariable, but not in multivariable analysis (Table 3).

The OS and PFS of the patients were assessed in three groups: no lymphatic disease, only pelvic-positive nodes and both pelvic- and paraaortic-positives nodes. For both DFS and OS, we found statistical differences between these groups with a $P$-value $<0.001$ (Figures 1 and 2).

\section{DISCUSSION}

The present study demonstrates the high incidence of pelvic and PALN involvement and the contribution of the SN biopsy to detect micrometastases in patients with locally advanced stages of cervical cancer.

A recent meta-analysis (Chemoradiotherapy for Cervical Cancer Meta-analysis Collaboration (CCCMAC), 2010)) including 13 trials has confirmed that the gold standard to treat patients with locally advanced-stages cervical cancer is CRC: a $6 \%$ improvement in 5 -year survival with chemoradiotherapy (hazard ratio $=0.81$, $P<0.001)$ was observed when chemoradiotherapy was compared with the same radiotherapy. However, this meta-analysis based on lymph node status, especially iliac node involvement, was not completed as there were insufficient data not allowing to state on the impact of lymphadenectomy. We observed a high incidence of pelvic and PALN involvement, $50 \%$ and $14 \%$, respectively, in our
Table 3 Univariable and multivariable analysis of potential predictive factors of pelvic or para-aortic lymph node metastasis in 42 patients with stage IB2-IIB cervical cancer

\begin{tabular}{lcc}
\hline & $\begin{array}{c}\text { Pelvic lymph node } \\
\text { metastases } \\
\text { Univariable analysis (P) }\end{array}$ & $\begin{array}{c}\text { Para-aortic lymph node } \\
\text { metastases } \\
\text { Univariable analysis (P) }\end{array}$ \\
\hline $\begin{array}{l}\text { Tumour size } \\
>30 \mathrm{~mm}\end{array}$ & 0.81 & 1 \\
$\begin{array}{l}\text { Tumour size } \\
>40 \mathrm{~mm}\end{array}$ & 0.38 & 0.88 \\
FIGO stage & 0.01 & 0.43 \\
Post-menopausal & 0.44 & 0.3 \\
status & 0.12 & 0.88 \\
Age & 0.15 & 1 \\
Histology & & 1 \\
\hline
\end{tabular}

Abbreviation: FIGO = International Federation of Gynecology and Obstetrics

study. However, despite a significant difference in both OS and PFS between patients with positive pelvic and paraaortic positive nodes, and patients with only positive pelvic nodes, we cannot conclude that lymphadenectomy has a therapeutic effect, as no difference was observed between patients with and without positive pelvic lymph nodes. This explains why some authors recommend paraaortic lymphadenectomy only (preferentially by retroperitoneal approach) to determine the extent of radiotherapy while limiting the side effects on small bowel, suggesting that adjuvant chemoradiation is able to sterilise all pelvic lymph nodes in patients with locally advanced-stages cervical cancer. However, Houvenaeghel et al (2006) found that $16 \%$ of women with locally advanced cervical cancer initially treated by CRC had persistent positive pelvic lymph nodes. In a series of 73 patients with IB2 - IIB cervical cancer treated by CRC, followed by paraaortic lymphadenectomy associated with pelvic lymphadenectomy or pelvic lymph node sampling in 36 patients, Morice et al (2007) reported that 13 patients $(36 \%)$ had persistent positive pelvic lymph node after CRC. In the study of Morice et al (2007), among the four pelvic lymph node relapses, three occurred in patients who had not undergone pelvic lymphadenectomy. Moreover, in a multivariate analysis, Rouzier et al (2005) demonstrated that, in addition to tumour size, the main determinant of pelvic relapse was pelvic lymph node involvement. Therefore, in addition to prognostic relevance, pelvic lymphadenectomy may have a therapeutic impact by reducing the risk of lymph node relapse, thus reinforcing the idea that when lymphadenectomy is indicated before CRC, both pelvic and paraaortic lymphadenectomy should be performed. Indeed, Marnitz et al (2005) showed that removal of positive pelvic and/or positive PALNs was associated with significant improvement in OS, confirming that lymphadenectomy should be performed before primary chemoradiation. Comparing survival of patients undergoing negative PALN identified by surgical staging to patients with only radiographic exclusion of PALN metastases, Gold et al (2008) showed that patients with radiographic evaluation only had a poorer prognosis supporting the therapeutic effect of lymphadenectomy. Moreover, Tseng et al (2010) built a nomogram in patients with locally advanced-stage cervical cancer, showing a high heterogeneity in predicting death, but underlined the preponderant impact of both pelvic and paraaortic involvement. Finally, our results underline that threequarters of patients with lymph node metastases were located in the pelvis, whereas only $20 \%$ had pelvic and paraaortic involvement, and only $6 \%$ had isolated paraaortic metastases. These data are of particular relevance, as two trials included in a recent metaanalysis (Lukka et al, 2002; Green et al, 2005; Chemoradiotherapy for Cervical Cancer Meta-analysis Collaboration (CCCMAC), 2010) showed greater benefits of adding chemotherapy after CRC, with 
an absolute improvement of $19 \%$ at 5 years. Patients with lymph node involvement, especially with pelvic and/or paraaortic metastases, could be good candidates for this new regimen.

Our SN detection is low compared with those observed in patients with early stages of cervical cancer (Plante et al, 2003; Martinez-Palones et al, 2004; Coutant et al, 2007), but concur with those of previous studies on SN in locally advanced stages of cervical cancer (Coutant et al, 2007). This difference in detection rates may be explained by the obstruction of lymphatic vessels by tumour embols. Moreover, we found a high false negative rate of $20 \%$ in the present study, contrasting with that of Altgassen et al (2009) reporting a false negative rate under $10 \%$ for tumour size below $2 \mathrm{~cm}$ for early stages of cervical cancer. All these considerations underline that SN procedure cannot be considered an alternative to lymphadenectomy in patients with locally advanced stages of cervical cancer. Despite a low SN detection and a high false negative rate, our results underline the contribution of ultrastaging, using combined serial sectioning and IHC to detect micrometastases; $20 \%$ of our patients with lymph node involvement were exclusively diagnosed as a result of ultrastaging. Lentz et al (2004), using IHC without serial sectioning, detected micrometastases in 19 out of a series of 132 women with 3106 negative lymph nodes on routine histology (15\%; 95\% confidence interval: 9-22\%). Silva et al (2005) confirmed the contribution of IHC in detecting micrometastases in 5 of 98 negative SNs. In a recent review on SN biopsy in cervical cancer, using H\&E and IHC (Lambaudie et al, 2003; Martinez-Palones et al, 2004; Niikura et al, 2004; Kraft et al, 2006) on SNs, no micrometastases were detected. Using $\mathrm{H} \& \mathrm{E}$, serial sectioning and IHC, the incidence of micrometastases ranged from $0 \%$ to $47.4 \%$ with a mean value of $28.3 \%$, similar to that observed in the current study.

From a clinical view point, Juretzka et al (2004) first underlined the potential prognostic relevance of micrometastases and recommended adjuvant therapy for these patients. In a casecontrol study, Marchiole et al (2005) found that the relative risk of recurrence in the presence of true micrometastases (focus of metastatic disease ranging from $0.2 \mathrm{~mm}$ to no more than $2 \mathrm{~mm}$ ) was 2.30 (confidence interval: $1.65-3.20, P<0.01$ ). Moreover, in series of 894 patients, Horn et al (2008) confirmed the prognostic relevance of detecting micrometastases with a correlation between their presence and the risk of recurrence. Hence, all these data reinforce the notion that patients with metastases, including those with micrometastases detected in SNs, could be candidates for adjuvant chemotherapy after CRC.

Some limitations of the present study have to be underlined. First, the retrospective nature of the study cannot exclude the risk of potential bias. Second, no difference was observed between patients with and without positive pelvic lymph nodes, raising the issue on the rational of systematic pelvic lymphadenectomy. However, this could be explained by both the sample size of the study and the relatively short follow-up with few events (seven recurrences). Concerning the rational for completion of the surgery in our protocol, it is clear that no consensus exists on its indication and on its impact on survival while exposing patients to the risk of potential severe postoperative complications. Third, despite the contribution of ultrastaging using combined serial sectioning and IHC to detect micrometastases, our study was unable to prove the therapeutic effect of pelvic lymphadenectomy. This could suggest that pre-therapeutic pelvic lymphadenectomy is unnecessary, as pelvic radiotherapy could be sufficiently effective on positive pelvic nodes. However, even in new regimens of radiotherapy or chemoradiation, there is a lack of data on pelvic node sterilisation, particularly, when using radiotherapy boost (Haie-Meder et al, 2009).

\section{CONCLUSION}

The SN procedure resulted in an increased detection rate of pelvic node metastases, which are often underestimated, despite a high false negative rate. According to a recent meta-analysis showing the benefits of adding chemotherapy after CRC in case of lymph node metastases, patients with lymph node metastases could be good candidates for this regimen. Further studies are required to evaluate whether pre-therapeutic node staging, including paraaortic and pelvic lymphanedectomy, should be performed in women with locally advanced cervical cancer.

\section{REFERENCES}

Altgassen C, Muller N, Hornemann A, Kavallaris A, Hornung D, Diedrich $\mathrm{K}$, Jarutat $\mathrm{T}$ (2009) Immunohistochemical workup of sentinel nodes in endometrial cancer improves diagnostic accuracy. Gynecol Oncol 114(2): $284-287$

Barranger E, Cortez A, Commo F, Marpeau O, Uzan S, Darai E, Callard P (2004a) Histopathological validation of the sentinel node concept in cervical cancer. Ann Oncol 15(6): 870-874

Barranger E, Cortez A, Uzan S, Callard P, Darai E (2004b) Value of intraoperative imprint cytology of sentinel nodes in patients with cervical cancer. Gynecol Oncol 94(1): 175-180

Barranger E, Grahek D, Cortez A, Talbot JN, Uzan S, Darai E (2003) Laparoscopic sentinel lymph node procedure using a combination of patent blue and radioisotope in women with cervical carcinoma. Cancer 97(12): $3003-3009$

Bezu C, Coutant C, Ballester M, Feron JG, Rouzier R, Uzan S, Darai E (2010) Ultrastaging of lymph node in uterine cancers. J Exp Clin Cancer Res 29: 5

Boss EA, Barentsz JO, Massuger LF, Boonstra H (2000) The role of MR imaging in invasive cervical carcinoma. Eur Radiol 10(2): 256-270

Chemoradiotherapy for Cervical Cancer Meta-analysis Collaboration (CCCMAC) (2010) Reducing uncertainties about the effects of chemoradiotherapy for cervical cancer: individual patient data meta-analysis. Cochrane Database Syst Rev (1): CD008285

Chu KK, Chang SD, Chen FP, Soong YK (1997) Laparoscopic surgical staging in cervical cancer - preliminary experience among Chinese. Gynecol Oncol 64(1): 49-53
Coutant C, Morel O, Delpech Y, Uzan S, Darai E, Barranger E (2007) Laparoscopic sentinel node biopsy in cervical cancer using a combined detection: 5-years experience. Ann Surg Oncol 14(8): 2392 - 2399

Gold MA, Tian C, Whitney CW, Rose PG, Lanciano R (2008) Surgical vs radiographic determination of para-aortic lymph node metastases before chemoradiation for locally advanced cervical carcinoma: a Gynecologic Oncology Group Study. Cancer 112(9): $1954-1963$

Green J, Kirwan J, Tierney J, Vale C, Symonds P, Fresco L, Williams C, Collingwood M (2005) Concomitant chemotherapy and radiation therapy for cancer of the uterine cervix. Cochrane Database Syst Rev (3): CD002225

Haie-Meder C, Morice P, Castiglione M (2009) Cervical cancer: ESMO clinical recommendations for diagnosis, treatment and follow-up. Ann Oncol 20(Suppl 4): 27-28

Hertel H, Kohler C, Elhawary T, Michels W, Possover M, Schneider A (2002) Laparoscopic staging compared with imaging techniques in the staging of advanced cervical cancer. Gynecol Oncol 87(1): 46-51

Horn LC, Hentschel B, Fischer U, Peter D, Bilek K (2008) Detection of micrometastases in pelvic lymph nodes in patients with carcinoma of the cervix uteri using step sectioning: Frequency, topographic distribution and prognostic impact. Gynecol Oncol 111(2): 276-281

Houvenaeghel G, Lelievre L, Rigouard AL, Buttarelli M, Jacquemier J, Viens P, Gonzague-Casabianca L (2006) Residual pelvic lymph node involvement after concomitant chemoradiation for locally advanced cervical cancer. Gynecol Oncol 102(1): 74-79 
Hricak H, Lacey CG, Sandles LG, Chang YC, Winkler ML, Stern JL (1988) Invasive cervical carcinoma: comparison of $\mathrm{MR}$ imaging and surgical findings. Radiology 166(3): 623-631

Juretzka MM, Jensen KC, Longacre TA, Teng NN, Husain A (2004) Detection of pelvic lymph node micrometastasis in stage IA2-IB2 cervical cancer by immunohistochemical analysis. Gynecol Oncol 93(1): 107-111

Kamelle SA, Rutledge TL, Tillmanns TD, Gould NS, Cohn DE, Wright J, Herzog TJ, Rader JS, Gold MA, Johnson GA, Walker JL, Mannel RS, McMeekin DS (2004) Surgical-pathological predictors of disease-free survival and risk groupings for IB2 cervical cancer: do the traditional models still apply? Gynecol Oncol 94(2): 249-255

Kim SH, Choi BI, Han JK, Kim HD, Lee HP, Kang SB, Lee JY, Han MC (1993) Preoperative staging of uterine cervical carcinoma: comparison of CT and MRI in 99 patients. J Comput Assist Tomogr 17(4): 633-640

Kim SH, Kim SC, Choi BI, Han MC (1994) Uterine cervical carcinoma: evaluation of pelvic lymph node metastasis with MR imaging. Radiology 190(3): $807-811$

Kraft O, Sevcik L, Klat J, Koliba P, Curik R, Kriozva H (2006) Detection of sentinel lymph nodes in cervical cancer. A comparison of two protocols. Nucl Med Rev Cent East Eur 9(1): 65-68

Lai CH, Huang KG, Hong JH, Lee CL, Chou HH, Chang TC, Hsueh S, Huang HJ, Ng KK, Tsai CS (2003) Randomized trial of surgical staging (extraperitoneal or laparoscopic) $v s$ clinical staging in locally advanced cervical cancer. Gynecol Oncol 89(1): 160-167

Lambaudie E, Collinet P, Narducci F, Sonoda Y, Papageorgiou T, Carpentier P, Leblanc E, Querleu D (2003) Laparoscopic identification of sentinel lymph nodes in early stage cervical cancer: prospective study using a combination of patent blue dye injection and technetium radiocolloid injection. Gynecol Oncol 89(1): $84-87$

Lanciano RM, Martz K, Coia LR, Hanks GE (1991) Tumor and treatment factors improving outcome in stage III-B cervix cancer. Int J Radiat Oncol Biol Phys 20(1): 95-100

Leblanc E, Narducci F, Frumovitz M, Lesoin A, Castelain B, Baranzelli MC Taieb S, Fournier C, Querleu D (2007) Therapeutic value of pretherapeutic extraperitoneal laparoscopic staging of locally advanced cervical carcinoma. Gynecol Oncol 105(2): 304-311

Lentz SE, Muderspach LI, Felix JC, Ye W, Groshen S, Amezcua CA (2004) Identification of micrometastases in histologically negative lymph nodes of early-stage cervical cancer patients. Obstet Gynecol 103(6): 1204-1210

Lukka H, Hirte H, Fyles A, Thomas G, Elit L, Johnston M, Fung MF, Browman G (2002) Concurrent cisplatin-based chemotherapy plus radiotherapy for cervical cancer - a meta-analysis. Clin Oncol ( $R$ Coll Radiol) 14(3): 203-212

Marchiole P, Buenerd A, Benchaib M, Nezhat K, Dargent D, Mathevet P (2005) Clinical significance of lympho vascular space involvement and lymph node micrometastases in early-stage cervical cancer: a retrospective case-control surgico-pathological study. Gynecol Oncol 97(3): $727-732$

Marnitz S, Kohler C, Roth C, Fuller J, Hinkelbein W, Schneider A (2005) Is there a benefit of pretreatment laparoscopic transperitoneal surgical staging in patients with advanced cervical cancer? Gynecol Oncol 99(3): 536-544

Martinez-Palones JM, Gil-Moreno A, Perez-Benavente MA, Roca I, Xercavins J (2004) Intraoperative sentinel node identification in early stage cervical cancer using a combination of radiolabeled albumin injection and isosulfan blue dye injection. Gynecol Oncol 92(3): 845-850
Michel G, Morice P, Castaigne D, Leblanc M, Rey A, Duvillard P (1998) Lymphatic spread in stage Ib and II cervical carcinoma: anatomy and surgical implications. Obstet Gynecol 91(3): 360-363

Morice P, Castaigne D (2005) Advances in the surgical management of invasive cervical cancer. Curr Opin Obstet Gynecol 17(1): 5-12

Morice P, Uzan C, Zafrani Y, Delpech Y, Gouy S, Haie-Meder C (2007) The role of surgery after chemoradiation therapy and brachytherapy for stage IB2/II cervical cancer. Gynecol Oncol 107(1 Suppl 1): S122-S124

Narayan K, McKenzie AF, Hicks RJ, Fisher R, Bernshaw D, Bau S (2003) Relation between FIGO stage, primary tumor volume, and presence of lymph node metastases in cervical cancer patients referred for radiotherapy. Int J Gynecol Cancer 13(5): 657-663

Niikura H, Okamura C, Akahira J, Takano T, Ito K, Okamura K, Yaegashi N (2004) Sentinel lymph node detection in early cervical cancer with combination 99mTc phytate and patent blue. Gynecol Oncol 94(2): 528-532

Petru E, Luck HJ, Stuart G, Gaffney D, Millan D, Vergote I (2009) Gynecologic Cancer Intergroup (GCIG) proposals for changes of the current FIGO staging system. Eur J Obstet Gynecol Reprod Biol 143(2): 69-74

Piver MS (1984) Extended field irradiation in the treatment of patients with cervical carcinoma involving biopsy proven para-aortic nodes. Int $J$ Radiat Oncol Biol Phys 10(10): 1993-1994

Plante M, Renaud MC, Tetu B, Harel F, Roy M (2003) Laparoscopic sentinel node mapping in early-stage cervical cancer. Gynecol Oncol 91(3): $494-503$

Rouzier R, Morice P, De Crevoisier R, Pomel C, Rey A, Bonnet K, RecoulesArche A, Duvillard P, Lhomme C, Haie-Meder C, Castaigne D (2005) Survival in cervix cancer patients treated with radiotherapy followed by radical surgery. Eur J Surg Oncol 31(4): 424-433

Selman TJ, Mann C, Zamora J, Appleyard TL, Khan K (2008) Diagnostic accuracy of tests for lymph node status in primary cervical cancer: a systematic review and meta-analysis. CMAJ 178(7): 855-862

Shepherd JH (1996) Cervical and vulva cancer: changes in FIGO definitions of staging. Br J Obstet Gynaecol 103(5): 405-406

Sheu MH, Chang CY, Wang JH, Yen MS (2001) Preoperative staging of cervical carcinoma with MR imaging: a reappraisal of diagnostic accuracy and pitfalls. Eur Radiol 11(9): 1828-1833

Silva LB, Silva-Filho AL, Traiman P, Triginelli SA, de Lima CF, Siqueira CF, Barroso A, Rossi TM, Pedrosa MS, Miranda D, Melo JR (2005) Sentinel node detection in cervical cancer with $(99 \mathrm{~m}) \mathrm{Tc}$-phytate. Gynecol Oncol 97(2): $588-595$

Stryker JA, Mortel R (2000) Survival following extended field irradiation in carcinoma of cervix metastatic to para-aortic lymph nodes. Gynecol Oncol 79(3): 399-405

Subak LL, Hricak H, Powell CB, Azizi L, Stern JL (1995) Cervical carcinoma: computed tomography and magnetic resonance imaging for preoperative staging. Obstet Gynecol 86(1): $43-50$

Tseng JY, Yen MS, Twu NF, Lai CR, Horng HC, Tseng CC, Chao KC, Juang CM (2010) Prognostic nomogram for overall survival in stage IIB-IVA cervical cancer patients treated with concurrent chemoradiotherapy. Am J Obstet Gynecol 202(2): 174.e1 - 174.e7

Vergote I, Amant F, Berteloot P, Van Gramberen M (2002) Laparoscopic lower para-aortic staging lymphadenectomy in stage IB2, II, and III cervical cancer. Int J Gynecol Cancer 12(1): 22-26

Zander J, Baltzer J, Lohe KJ, Ober KG, Kaufmann C (1981) Carcinoma of the cervix: an attempt to individualize treatment. Results of a 20-year cooperative study. Am J Obstet Gynecol 139(7): $752-759$

This work is published under the standard license to publish agreement. After 12 months the work will become freely available and the license terms will switch to a Creative Commons Attribution-NonCommercial-Share Alike 3.0 Unported License. 